\title{
ATIVIDADE ANTIMICROBIANA DE FLAVONOIDES: UMA REVISÃO DE LITERATURA
}

\author{
ANTIMICROBIAL ACTIVITY OF FLAVONOIDS: A LITERATURE REVIEW
}

\author{
Andressa Dias da Silva ${ }^{1}$, Layza Kowalski ${ }^{1}$, Andressa Rodrigues Pagno ${ }^{1}$, Mariana \\ Piana $^{1}$ \\ ${ }^{1}$ Universidade Regional Integrada do Alto Uruguai e das Missóes, URI; Santo Ângelo, RS, \\ Brasil.
}

\section{RESUMO}

Plantas são, geralmente, uma rica fonte de constituintes ativos que fornecem benefícios medicinais ou de prevenção contra diversas doenças e enfermidades, por conseguinte, levando em consideração o preocupante aumento na taxa de infecção por micro-organismos resistentes aos antibióticos, tem-se estimulado a procura por compostos que possuam potencial antimicrobiano, e os flavonoides possuem grandes atividades biológicas e terapêuticas que se encaixam nessa categoria. O presente estudo objetivou reunir dados sobre a atividade antimicrobiana dos flavonoides, e assim apresentar os benefícios do uso de plantas medicinais com estes compostos em infecçóes por bactérias. Trata-se de uma revisão de literatura, realizada nas bases de dados Scientific Electronic Library Online, US National Library of Medicine National Institutes of Health e Science Direct, de artigos científicos publicados entre 2005 e 2020. Os flavonoides podem ser capazes de inibir o crescimento bacteriano, através de diferentes mecanismos, como a alteração da permeabilidade de membrana e parede celular, inibição da síntese de ácidos nucléicos, neutralização dos fatores de virulência, inibição de urease e também pela atividade sinérgica com antibióticos. O desenvolvimento de novos medicamentos antimicrobianos torna-se cada vez mais necessário, inclusive fitoterápicos, o que torna os flavonoides como candidatos promissores.

Descritores: flavonoides; anti-infecciosos; plantas.

\section{ABSTRACT}

Plants are generally a rich source of active constituents that provide medicinal or preventive benefits against various diseases and illnesses, therefore, taking into account the worrying increase in the rate of infection by antibiotic-resistant microorganisms, it has been stimulated to looking for compounds that have antimicrobial potential, and flavonoids have great biological and therapeutic activities that fall into this category. The present study aimed to gather data on the antimicrobial activity of flavonoids, and thus present the benefits of using medicinal plants with these compounds in infections by bacteria. This is a literature review, carried out in the Scientific Electronic Library Online, US National Library of Medicine National Institutes of Health and Science Direct databases, of scientific articles published between 2005 and 2020. Flavonoids may be able to inhibit growth bacterial, through different mechanisms, such as alteration of the membrane and cell wall permeability, inhibition of 
nucleic acid synthesis, neutralization of virulence factors, inhibition of urease, and also by synergistic activity with antibiotics. The development of new antimicrobial drugs is becoming more and more necessary, including herbal medicines, which makes flavonoids as promising candidates.

Descriptors: flavonoids; anti-infective agents; plants.

\section{INTRODUÇÃO}

Os produtos naturais, especialmente os derivados de plantas, têm sido utilizados para auxiliar a humanidade na manutenção da saúde e na cura de doenças desde o início da medicina ${ }^{1}$. Do ponto de vista farmacêutico, plantas com uma longa história de uso medicinal são, geralmente, uma rica fonte de constituintes ativos que fornecem benefícios medicinais ou de prevenção contra diversas doenças e enfermidades ${ }^{2}$.

Diversos fitoquímicos encontrados em variadas espécies têm sido descobertos e se tornado essenciais para o desenvolvimento de novos produtos farmacêuticos ${ }^{3}$. A relevância dos princípios ativos das plantas tem reforçado significativamente o interesse em pesquisa nas atividades biológicas dessas substâncias que podem ser utilizadas como agentes medicinais ${ }^{1,3}$.

Simultaneamente, relatórios mundiais têm notificado o preocupante aumento na taxa de micro-organismos resistentes aos antibióticos, como é o caso da Escherichia coli e Klebsiella pneumoniae com as cefalosporinas de terceira geração, que apresentam uma taxa de até $48 \%$ e $71 \%$ de resistência, respectivamente, no continente americano. Tal crescimento tem estimulado a procura por compostos que possuam potencial antimicrobiano em vegetais ${ }^{4,5}$.

A investigação de plantas medicinais que apresentam substâncias capazes de agir contra micro-organismos patógenos torna-se de grande importância, ao passo que, o aumento da resistência bacteriana aos antibióticos mostra-se como um problema que afeta a população em geral, representando um desafio para o manejo clínico, maior mortalidade por infecçôes e maior custo de tratamento Gamong the plant species identified, nearly ten thousand has any known medicinal property. In the Araripe Plateau highlights the use of Annona muricata Linnaeus (Soursop. Desta forma, o uso de plantas com potencial antimicrobiano tem sido empregado como alternativas naturais para o tratamento ou prevenção de diversas enfermidades 7 , principalmente nos países em desenvolvimento que possuem escassos recursos financeiros.

Dentre os compostos naturais atualmente estudados, destacam-se os flavonoides em virtude de sua ampla variedade de atividades biológicas e terapêuticas, demonstradas tanto in vitro como in vivo. Sendo esta a família de compostos alvo de muitas pesquisas relacionadas a atividade antibacteriana. Desta forma, o objetivo do presente trabalho é fazer uma revisão bibliográfica da atividade antimicrobiana dos flavonoides, de forma a 
apresentar os benefícios do uso de plantas medicinais com estes compostos em infecçóes por bactérias.

\section{METODOLOGIA}

Este estudo constitui-se de uma revisão da literatura, incluindo a análise de artigos científicos mais relevantes publicados, entre os anos de 2005 a 2020. A busca pelos artigos foi realizada nas seguintes bases de dados: Scientific Electronic Library Online (SciELO), US National Library of Medicine National Institutes of Health (PUBMED) e Science Direct. Os descritores utilizados, em língua portuguesa e inglesa, foram: flavonoides, antimicrobiano, plantas, atividade (DeCS - Descritores em Ciências da Saúde). Os artigos foram selecionados de acordo com o título e o resumo, onde se destacava o tema do estudo.

\section{REVISÁO}

\section{A resistência microbiana}

A resistência microbiana ocorre quando micro-organismos (bactérias, fungos, vírus ou parasitas) alteram suas características de patogenicidade quando expostos a medicamentos antimicrobianos (antibióticos, antifúngicos, antivirais, antimaláricos e anti-helmínticos). Os micro-organismos que desenvolvem resistência microbiana sáo muitas vezes chamados de "superbactérias", como resultado disso, os medicamentos tornam-se ineficazes, fazendo com que as infecções persistam no indivíduo afetado, aumentando o risco de disseminaçáo para outras pessoas. Essa resistência é acelerada pelo mau uso e uso excessivo de antibióticos, bem como, pela prevençáo e controle deficientes das infecçóes ${ }^{8}$.

A resistência pode se dar por meio de características intrínsecas do microorganismo ou adquirida via transmissão de material genético. Esta, se divide nos mecanismos de produção de enzimas inativadoras de antibióticos, mudança no sítio de ação do antibiótico e criação de novas vias metabólicas e a exclusão do antibiótico via porinas (Gram-negativos) ou bombas de efluxo ?.

Pacientes com infecçóes geradas por bactérias resistentes a medicamentos apresentam maior risco de piores desfechos clínicos e morte, além disso, aumenta o custo dos cuidados de saúde com estadias prolongadas nos hospitais e exige maiores cuidados intensivos ${ }^{8}$.

Os micro-organismos gram-negativos são alvo de particular preocupação pelo fato de estarem se tornando resistentes a quase todas as opçóes de medicamentos disponíveis 
${ }^{10}$, tornando-os bacilos MDR (multidroga resistentes). As infecçôes mais graves ocorrem em centros de tratamento de saúde, sendo comumente causadas por enterobactérias (principalmente Klebsiella pneumoniae), Pseudomonas aeruginosa e Acinetobacter ${ }^{11,12}$.

A resistência a Klebsiella pneumoniae espalhou-se mundialmente, sendo hoje uma das principais causas de infecções hospitalares (como pneumonia, infecções da corrente sanguínea, infecçóes em recém-nascidos e pacientes de unidade de terapia intensiva). Em alguns países, os antibióticos carbapenêmicos não demonstram eficácia em mais da metade das pessoas tratadas por infecçóes contra esse patógeno ${ }^{4}$.

Entre os patógenos Gram-positivos, a pandemia global de espécies resistentes de Staphylococcus aureus e Enterococcus representa a maior ameaça. Estima-se que pessoas infectadas com MRSA (Staphylococcus aureus resistente à meticilina) tenham probabilidade 64\% maior de morrer do que pessoas com uma forma não resistente de infecção. A cada ano MRSA mata mais americanos do que o HIV/AIDS, a doença de Parkinson, o enfisema pulmonar e os homicídios juntos ${ }^{10,13}$. O aumento global da resistência a medicamentos entre patógenos respiratórios comum, incluindo Streptococcus pneumoniae e Mycobacterium tuberculosis, é epidêmica ${ }^{11}$.

A colistina é o último recurso no tratamento de infecçóes fatais causadas por enterobactérias resistentes aos carbapenêmicos. A resistência a ela foi detectada em vários países, tornando as infecçóes causadas por essas bactérias intratáveis ${ }^{13}$.

\section{O uso de plantas como alternativa a antibióticos}

$\mathrm{O}$ aumento da resistência microbiana aos antimicrobianos existentes é um sério e crescente problema de saúde ${ }^{14}$. Desse modo, há necessidade de buscar novas classes de fármacos antibacterianos, principalmente a partir de fontes naturais, que são uma boa alternativa ${ }^{15}$.

Ao longo dos anos, a eficácia das plantas no tratamento de doenças infecciosas sugere que os micro-organismos possuam uma capacidade reduzida de se adaptar ao mecanismo de ação antimicrobiano à base de plantas ${ }^{16,17}$. Essa capacidade reduzida pode ser devido à diferentes metabólitos secundários produzidos pelas plantas exercerem um papel na defesa de seu próprio metabolismo, resultando em uma maior atividade dessa planta tanto contra patógenos prejudiciais a ela quanto contra patógenos relacionados aos humanos ${ }^{18}$. Adicionalmente, o sinergismo gerado pelos vários compostos presentes em um único extrato pode ser atribuído aos diversos alvos que esses constituintes podem atuar, como receptores, enzimas, canais iônicos, anticorpos e, proteínas de transporte ${ }^{19}$.

Em consonância, o uso de plantas medicinais tem vantagens para a terapia antimicrobiana, como a reduçáo dos efeitos colaterais, melhor tolerância do paciente, maior economia, boa aceitação, e por ser fonte renovável, estar disponível na natureza ${ }^{20}$. 
Além disso, enquanto não surgem novos antimicrobianos com capacidade de tratar bactérias resistentes, a terapia sinérgica entre antimicrobianos conhecidos e extratos de plantas medicinais é uma boa alternativa de tratamento ${ }^{15}$. Segundo Chanda e Rakholiya ${ }^{21}$, a terapia combinada com extratos de plantas e antibióticos pode expandir o espectro antimicrobiano, evitar o aparecimento de resistência e diminuir a toxicidade.

\section{Flavonoides}

Flavonoides pertencem a um grupo de compostos orgânicos heterocíclicos, que estão presentes em diversas plantas e seus produtos relativos, como por exemplo, mel e própolis ${ }^{22}$. Diversas formas farmacêuticas (infusóes, bálsamos, cataplasmas) contendo flavonoides como constituintes ativos foram usadas durante séculos. Dentre seus usos tradicionais estão o tratamento e prevenção de doenças infecciosas e mediadas por feridas, por exemplo ${ }^{23}$; acne, infecçóes respiratórias ${ }^{24}$; doenças do trato gastrointestinal ${ }^{25}$; e do trato urinário ${ }^{26}$.

Quimicamente constituem-se como uma família de moléculas diversas que apresentam dois anéis aromáticos (A e B), que estão conectados por um anel pirano (C) (figura 1). Estes podem ser divididos em seis subgrupos: flavonas (apigenina), flavonóis (quercetina), catequinas (epicatequina), flavononas (hesperitina), antocianinas (cianina) e isoflavonas (genisteina) ${ }^{27}$.

Figura 1- Estrutura básica dos compostos flavonoides

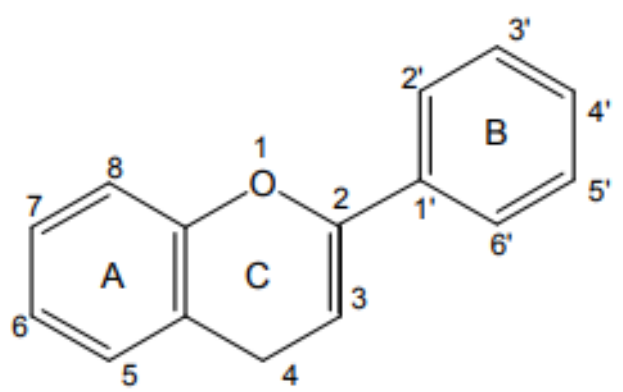

Fonte: HUBER, 200728 .

São sintetizados pela via metabólica do ácido chiquímico e ácido acético, a partir da fenilalanina. Primeiro, a fenilalanina é transformada em ácido cinâmico pela ação da enzima fenilalanina amônio liase. $\mathrm{O}$ ácido cinâmico é hidrolisado a ácido cumárico que é condensado a 3 unidades de malonil-CoA, que forma uma chalcona, a partir da qual todos os flavonoides são formados ${ }^{29}$. Esta família de compostos é alvo de muitas pesquisas antibacterianas. Há 14 classes de flavonoides, os quais são diferenciados com base em sua natureza química e posição de substituintes nos anéis $\mathrm{A}, \mathrm{B}$ e $\mathrm{C}^{30}$. As estruturas químicas das seis classes mais importantes são mostradas na figura 2. O grande número de relatos 
afirmando que os flavonoides possuem propriedades antimicrobianas pode ser atribuído a essas seis estruturas e seus derivados isoflavonoides ${ }^{23}$.

Os flavonoides podem ser isolados por técnicas cromatográficas e podem ser identificados por espectrofotometria de massa e ressonância magnética nuclear ${ }^{31}$.

Figura 2 - Estruturas das seis principais classes de flavonoides antibacterianos (flavonas, flavonóis, flavanonas, chalconas, flavan-3-ols).<smiles>O=c1cc(-c2ccccc2)oc2ccccc12</smiles>

Flavone<smiles>O=C1CCOc2ccccc21</smiles>

Flavanone<smiles>O=C(/C=C/c1ccccc1)c1ccccc1</smiles>

Chalcone

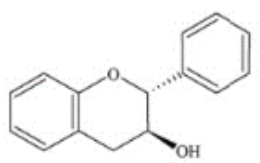

Flavan-3-ol (also known as catechin)<smiles>O=c1c(O)c(-c2ccccc2)oc2ccccc12</smiles>

Flavonol

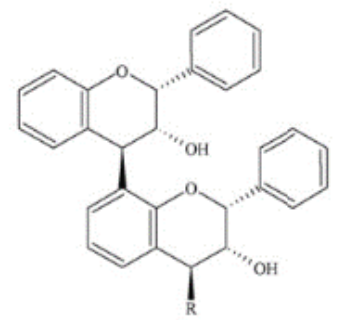

Proanthocyanidin (also known as flavolan or condensed tannin)

Fonte: CUSHNIE, $2005^{23}$.

Os flavonoides podem ser capazes de inibir o crescimento bacteriano, através de diferentes mecanismos, os quais são descritos a seguir:

\section{Alteraçáo da permeabilidade de membrana e parede celular}

A membrana plasmática bacteriana é responsável pela osmorregulação, respiração e processos de transporte, entre outras funçóes importantes. Para executar todas estas funções a membrana deve ser manter íntegra, pois, sua ruptura pode causar disfunção metabólica e levar as bactérias à morte ${ }^{32}$. $\mathrm{O}$ mecanismo de ação baseado na alteração da membrana refere-se à capacidade de danificar as estruturas da membrana celular ou afetar sua permeabilidade ${ }^{33}$.

A capacidade dos flavonoides de interagir com as membranas biológicas devido a sua lipofilicidade são fatores essenciais para sua atividade farmacológica. Muitos flavonoides contêm grupos hidroxila, que são responsáveis por conferir alguma polaridade 
e propriedades ácidas fracas à molécula ${ }^{34}$. A interação com a bicamada lipídica depende do $\mathrm{pH}$ que determina as cargas eletrostáticas dos flavonoides e dos lipídios. Geralmente, o pH baixo resulta em menor desprotonaçáo de grupos polares e, isso ocasiona em uma penetração mais profunda dos flavonoides na camada lipídica ${ }^{35}$.

A atividade dos flavonoides por ser evidenciada pela diminuição da fluidez da membrana celular e pela alteração da troca de nutrientes e metabólitos da célula, inibindo o fornecimento de energia para a célula bacteriana ${ }^{36}$.

\section{Inibição da síntese de ácidos nucléicos}

Os flavonoides podem reduzir a síntese de ácido desoxirribonucleico (DNA) e ácido ribonucleico (RNA) bacteriano através da inibição da topoisomerase ${ }^{37,38}$. Conjugados fluorados de chalconas-1,2,3-triazóis com atividade antimicrobiana promissora foram relatados por interagir com a topoisomerase através de várias interaçôes não covalentes 39. Além disso, estudos de modelagem molecular indicam que compostos flavonoides sintéticos inibem a atividade da DNA girase, ligando-se por pontes de hidrogênio aos sítios ativos da DNA girase, confirmando sua atividade antimicrobiana ${ }^{40}$.

Estudo realizado por Mori e colaboradores ${ }^{41}$ mostrou que a síntese de DNA pode ser fortemente inibida em Proteusvulgaris, e que a síntese de RNA é mais afetada em Staphylococcus aureus. Nesse mesmo estudo, a síntese de proteínas e lipídeos também sofreu uma leve alteração. Esse efeito, segundo os autores, é atribuído ao possível fato de que o anel B dos flavonoides pode desempenhar um importante papel na intercalação ou ligação de hidrogênio com o empilhamento de bases dos ácidos nucléicos e que isso pode explicar a inibiçáo na síntese de DNA e RNA.

Estudos realizados por Ayman e colaboradores mostraram boa atividade antimicrobiana de uma nova série de chalconas de ditiocarbamato pelaligaçáo tanto para o DNA, quanto para a PEA transferase (fosfoetanolaminatransferase envolvida no mecanismo de resistência) contra Pseudomonas aeruginosa e cepas resistentes à Klebsiella pneumoniae ${ }^{42}$.

\section{Neutralizaçáo dos fatores de virulência}

As toxinas bacterianas desempenham um papel fundamental na patogênese das bactérias, podendo a levar a doença fatal mesmo após sua morte ${ }^{43}$. Estudos mostram que flavonoides podem neutralizar fatores de virulência de bactérias deixando as células humanas protegidas ${ }^{44,45}$.

Choi e colaboradores comprovaram que a catequina polimerizada bloqueia o efeito da toxina do Staphyloccocus aureus, tanto in vitro como in vivo ${ }^{46}$. 


\section{Inibição de Urease}

A Helicobacter pylori (H. pylori), microrganismo conhecido por causar infecções gástricas, secreta urease durante seus estágios de infecção para sobreviver ao baixo $\mathrm{pH}$ do estômago ${ }^{23}$. Estudos sugerem que classes de isoflavonas e chalconas inibem esta enzima. Isso pode ser um possível argumento para explicar a atividade bem-sucedida do flavonol quercetina testado na Guiné em suínos ${ }^{47}$, bem como, a eficácia clínica de derivados de chalconas no tratamento da infecção humana por $H$. pylori ${ }^{48}$.

Outros efeitos também podem ser atribuídos a esta atividade, como a neutralização de genes desta bactéria como o VacA e, também é possível que alguns flavonoides tenham atividade direta contra $H$. pyloriou tenham efeito sinérgico quando usados com antibióticos contra esse microrganismo ${ }^{23}$.

\section{Atividade sinérgica com antibióticos}

Há muitos relatos de que os flavonoides aumentam a atividade dos antibióticos, reduzindo a concentração inibitória mínima (CIM) dos antibióticos em diferentes bactérias ${ }^{23}$, sendo as informaçóes sobre algumas das combinaçóes mais potentes apresentadas na tabela 1. Pode-se observar, por exemplo, que a epicatequina reduz a CIM dos antibióticos 邓-lactâmicos contra MRSA mais de 512 vezes ${ }^{23}$.

Tabela 1-Resposta sinérgica gerada pela combinação de flavonoides e antibióticos.

\begin{tabular}{|c|c|c|c|c|}
\hline Flavonoide & $\begin{array}{l}\text { Antibiótico/ } \\
\text { classe }\end{array}$ & $\begin{array}{l}\text { Microrganismo } \\
\text { testado }\end{array}$ & $\begin{array}{l}\text { CIM de ATB } \\
\text { reduzida }\end{array}$ & Referência \\
\hline Epicatequina & Oxacicilina & MRSA & $256-512$ & $\begin{array}{l}\text { BERNAL, ZLOH } \\
\text { \&TAYLOR, } 20099^{49}\end{array}$ \\
\hline Quercetina & $\beta$-Lactâmicos & $\begin{array}{l}\text { S. aureus resistente } \\
\text { à penicilina }\end{array}$ & $\geq 20-\geq 80$ & $\begin{array}{l}\text { EUMKEB et al, } \\
2010^{50}\end{array}$ \\
\hline Miricetina & Isoniazida & $\begin{array}{l}\text { Mycobacterium } \\
\text { spp. }\end{array}$ & $8-16$ & $\begin{array}{l}\text { LECHNER, } \\
\text { GIBBONS \& } \\
\text { BUCAR, } 2008^{51}\end{array}$ \\
\hline Apigenina & $\begin{array}{l}\text { Ampicilina } \\
\text { Ceftriaxona }\end{array}$ & MRSA & $\begin{array}{l}800 \text { para } 107 \mu \mathrm{g} / \mathrm{ml} \\
58 \text { para } 2,6 \mu \mathrm{g} / \mathrm{ml}\end{array}$ & $\begin{array}{c}\text { AKILANDESWARI } \\
\text { \& RUCKMANI, } \\
2016^{52}\end{array}$ \\
\hline Baicaleina & Penicilina & PPSA & $\begin{array}{c}64 \mathrm{~g} / \mathrm{mL} \text { para } 16,8 \\
\mathrm{~g} / \mathrm{mL}\end{array}$ & QIAN et al., $2015^{53}$ \\
\hline Luteolina & Ampicilina & MRSA & 128 para $64 \mu \mathrm{g} / \mathrm{mL}$ & $\underset{54}{\text { USMAN et al.,2016 }}$ \\
\hline & Imipenem & & 516 para $417 \mu \mathrm{g} / \mathrm{mL}$ & \\
\hline
\end{tabular}

PPSA: S. aureus produtor de penicilase; MRSA: S. aureus resistente à meticilina. 
Os flavonoides quando utilizados em combinação com antibióticos aumentam sua atividade contra diversas bactérias. $\mathrm{Na}$ grande maioria das pesquisas realizadas, a combinação é aditiva ${ }^{55}$.

Estudos mostram que a atividade sinérgica náo é atribuída a apenas um composto, mas sim que diferentes compostos utilizados contribuem para esse efeito, em maior e em menor extensão, portanto o efeito é multi-objetivo. Tem sido relatado que os compostos fenólicos têm a capacidade de quebrar a estrutura da membrana citoplasmática, ocasionando em perda de sua integridade e posterior morte celular. Em concentraçóes subinibitórias, esses compostos (entre eles, os flavonoides) facilitariam a entrada do antibiótico no citoplasma da célula, facilitando assim a entrada de fluoroquinolonas, tetraciclina e cloranfenicol, que têm ação no interior da célula bacteriana, sendo assim, necessário o uso de menores doses de antibiótico ${ }^{56,57}$

\section{CONCLUSÓES}

Dados da Organização Mundial da Saúde (OMS) ${ }^{58}$ já mostraram que mecanismos de resistência estáo surgindo e se espalhando globalmente cada vez mais rápido, colocando em risco a capacidade humana de tratar doenças infecciosas comuns, ao ponto de um simples ferimento voltar a causar mortes. Ao passo que a resistência bacteriana avança o arsenal terapêutico disponível não acompanha esse aumento.

É sabido que os compostos fitoterápicos são menos potentes quando comparados com agentes tradicionais de tratamento, como por exemplo, os antibióticos. Porém, o desenvolvimento de novos medicamentos antimicrobianos torna-se cada vez mais necessário, e os flavonoides mostram-se como candidatos promissores. Pesquisas destes compostos e posteriores alteraçóes em sua estrutura podem permitir que sejam desenvolvidos novos fármacos antimicrobianos com boa atividade farmacológica para serem utilizados. Espera-se que através da compreensão dos mecanismos de ação dos flavonoides aqui relatados, tenha-se contribuído para isso.

\section{REFERÊNCIAS}

1. Moghadamtousi SZ, Fadaeinasab M, Nikzad S, Mohan G, Ali HM, Kadir HA. Annona muricata (Annonaceae): A review of its traditional uses, isolated acetogenins and biological activities. Int J Mol Sci. 2015;16(7):15625-58.

2. Ekor M. The growing use of herbal medicines: issues relating to adverse reactions and challenges in monitoring safety. Front Pharmacol [Internet]. 2014;4. Disponível em: http://journal.frontiersin.org/article/10.3389/fphar.2013.00177/abstract 
3. Pan S-Y, Zhou S-F, Gao S-H, Yu Z-L, Zhang S-F, Tang M-K, et al. New Perspectives on How to Discover Drugs from Herbal Medicines: CAM's Outstanding Contribution to Modern Therapeutics. Evidence-Based Complement Altern Med [Internet]. 2013;2013:1-25. Disponível em: http://www.hindawi.com/journals/ ecam/2013/627375/

4. World Health Organization - Global Report on Surveillance. Antimicrobial resistance [Internet]. Geneva; 2014 [citado 25 de junho de 2020]. Disponível em: https://www.who.int/health-topics/antimicrobial-resistance

5. Mujeeb F, Bajpai P, Pathak N. Phytochemical Evaluation, Antimicrobial Activity, and Determination of Bioactive Components from Leaves of Aegle marmelos. Biomed Res Int [Internet]. 2014;2014:1-11. Disponível em: http://www.hindawi.com/journals/ bmri/2014/497606/

6. Beneval Bento E, Brito Monteiro Á, Santiago Lemos IC, de Brito FE, de Oliveira DR, Alencar de Menezes IR, et al. Ethnopharmacological comparative study in the region of the Araripe of Annona muricata L. (Graviola). Rev Cuba Plantas Med. 2016;21(1):9-19.

7. Mithun Pai BH, Rajesh G, Shenoy R, Rao A. Anti-microbial efficacy of Soursop leaf extract (Annona muricata) on oral pathogens: An in-vitro study. J Clin Diagnostic Res. 2016;10(11):ZC01-4.

8. World Health Organization. Antimicrobial resistance [Internet]. 2018 [citado 25 de junho de 2020]. Disponível em: https://www.who.int/news-room/fact-sheets/detail/ antimicrobial-resistance

9. Adriano Queiroz de Mesquita. Mecanismos de Resistência a Antimicrobianos. Universidade Federal De Goiás; 2011.

10. Golkar Z, Bagasra O, Pace DG. Bacteriophage therapy: a potential solution for the antibiotic resistance crisis. J Infect Dev Ctries [Internet]. 13 de fevereiro de 2014;8(02):129-36. Disponível em: https://jidc.org/index.php/journal/article/ view/24518621

11. CDC - Center for Disease Control and Prevention. Antibiotic/Antimicrobial Resistance [Internet]. 2013 [citado 11 de fevereiro de 2020]. Disponível em: https://www.cdc.gov/drugresistance/biggest-threats.html?CDC_AA_ refVal=https\%3A\%2F\%2Fwww.cdc.gov\%2Fdrugresistance\%2Fbiggest_threats.html

12. Rossolini GM, Arena F, Pecile P, Pollini S. Update on the antibiotic resistance crisis. Curr Opin Pharmacol [Internet]. outubro de 2014;18:56-60. Disponível em: https:// linkinghub.elsevier.com/retrieve/pii/S1471489214001052

13. Gross M. Antibiotics in crisis. Curr Biol [Internet]. dezembro de 2013;23(24):R1063-5. Disponível em: https://linkinghub.elsevier.com/retrieve/pii/ S0960982213015121 
14. Davies J, Davies D. Origins and Evolution of Antibiotic Resistance. Microbiol Mol Biol Rev [Internet]. 1 de setembro de 2010;74(3):417-33. Disponível em: http:// mmbr.asm.org/cgi/doi/10.1128/MMBR.00016-10

15. Nascimento GGF, Locatelli J, Freitas PC, Silva GL. Antibacterial activity of plant extracts and phytochemicals on antibiotic-resistant bacteria. Brazilian J Microbiol [Internet]. outubro de 2000;31(4). Disponível em: http://www.scielo.br/scielo. php?script=sci_arttext\&pid=S1517-83822000000400003\&lng=en\&nrm=iso\&tlng=en

16. Cos P, Vlietinck AJ, Berghe D Vanden, Maes L. Anti-infective potential of natural products: How to develop a stronger in vitro 'proof-of-concept'. J Ethnopharmacol [Internet]. julho de 2006;106(3):290-302. Disponível em: https://linkinghub.elsevier. $\mathrm{com} /$ retrieve/pii/S0378874106001851

17. Gupta PD, Birdi TJ. Development of botanicals to combat antibiotic resistance. J Ayurveda Integr Med [Internet]. outubro de 2017;8(4):266-75. Disponível em: https://linkinghub.elsevier.com/retrieve/pii/S097594761630448X

18. Lila MA, Raskin I. Health-related Interactions of Phytochemicals. J Food Sci. 2005;70(3):54-66.

19. Wagner H, Ulrich-Merzenich G. Synergy research: Approaching a new generation of phytopharmaceuticals. Phytomedicine [Internet]. março de 2009;16(2-3):97-110. Disponível em: https://linkinghub.elsevier.com/retrieve/pii/S0944711308002559

20. Yuan H, Ma Q, Ye L, Piao G. The Traditional Medicine and Modern Medicine from Natural Products. Molecules [Internet]. 29 de abril de 2016;21(5):559.

Disponível em: http://www.mdpi.com/1420-3049/21/5/559

21. Chanda S, Rakholiya K. Combination therapy : Synergism between natural plant extracts and antibiotics against infectious diseases. Formatex. 2011;(January 2011):520-9.

22. Havsteen BH. The biochemistry and medical significance of the flavonoids. Pharmacol Ther [Internet]. novembro de 2002;96(2-3):67-202. Disponível em: https://linkinghub.elsevier.com/retrieve/pii/S016372580200298X

23. Cushnie TPT, Lamb AJ. Antimicrobial activity of flavonoids. Int J Antimicrob Agents. 2005;26(5):343-56.

24. Gutiérrez RMP, Mitchell S, Solis RV. Psidium guajava: A review of its traditional uses, phytochemistry and pharmacology. J Ethnopharmacol [Internet]. abril de 2008;117(1):1-27. Disponível em: https://linkinghub.elsevier.com/retrieve/pii/ S0378874108000536

25. Shan B, Cai Y-Z, Brooks JD, Corke H. The in vitro antibacterial activity of dietary spice and medicinal herb extracts. Int J Food Microbiol [Internet]. junho de 
2007;117(1):112-9. Disponível em: https://linkinghub.elsevier.com/retrieve/pii/ S0168160507001778

26. Nguyen HA, Denis O, Vergison A, Tulkens PM, Struelens MJ, Van Bambeke F. Intracellular Activity of Antibiotics in a Model of Human THP-1 Macrophages Infected by a Staphylococcus aureus Small-Colony Variant Strain Isolated from a Cystic Fibrosis Patient: Study of Antibiotic Combinations. Antimicrob Agents Chemother [Internet]. 1 de abril de 2009;53(4):1443-9. Disponível em: http://aac.asm.org/cgi/ doi/10.1128/AAC.01146-08

27. Hoffmann-Ribani R, Rodriguez-Amaya DB. Otimização de método para determinação de flavonóis e flavonas em frutas por cromatografia líquida de alta eficiência utilizando delineamento estatístico e análise de superfície de resposta. Quim Nova [Internet]. 2008;31(6):1378-84. Disponível em: http://www.scielo.br/scielo. php?script=sci_arttext \&pid=S0100-40422008000600020\&lng=pt \&nrm=iso\&tlng=pt

28. Senger Huber L. Flavonóides: Identificação De Fontes Brasileiras E Investigação Dos Fatores Responsáveis Pelas Variações Na Composição. Universidade Estadual de Campinas; 2007.

29. Kühnau J. The Flavonoids. A Class of Semi-Essential Food Components: Their Role in Human Nutrition. In: GH B, organizador. World Review of Nutrition and Dietetics [Internet]. 24º ed 1976. p. 117-91. Disponível em: https://www.karger.com/ Article/FullText/399407

30. Hendrich AB. Flavonoid-membrane interactions: possible consequences for biological effects of some polyphenolic compounds1. Acta Pharmacol Sin [Internet]. janeiro de 2006;27(1):27-40. Disponível em: http:/www.nature.com/ doifinder/10.1111/j.1745-7254.2006.00238.x

31. Flambó DFALP. Atividades biológicas dos flavonoides: atividade antimicrobiana. Universidade Fernando Pessoa - Faculdade de Ciências da Saúde. Tese de Mestrado. Universidade Fernando Pessoa; 2013.

32. Hartmann M, Berditsch M, Hawecker J, Ardakani MF, Gerthsen D, Ulrich AS. Damage of the Bacterial Cell Envelope by Antimicrobial Peptides Gramicidin S and PGLa as Revealed by Transmission and Scanning Electron Microscopy. Antimicrob Agents Chemother [Internet]. 1 de agosto de 2010;54(8):3132-42. Disponível em: http://aac.asm.org/cgi/doi/10.1128/AAC.00124-10

33. Babii C, Mihalache G, Bahrin LG, Neagu A-N, Gostin I, Mihai CT, et al. A novel synthetic flavonoid with potent antibacterial properties: In vitro activity and proposed mode of action. Virolle M-J, organizador. PLoS One [Internet]. 4 de abril de 2018;13(4):e0194898. Disponível em: https://dx.plos.org/10.1371/journal. pone. 0194898 
34. SMale ST, Tarakhovsky A, Natoli G. Chromatin contributions to the regulation of innate immunity. Annu Rev Immunol. 2014;32:489-511.

35. Movileanu L, Neagoe I, Flonta ML. Interaction of the antioxidant flavonoid quercetin with planar lipid bilayers. Int J Pharm [Internet]. setembro de 2000;205(1-2):135-46. Disponível em: https://linkinghub.elsevier.com/retrieve/pii/ S0378517300005032

36. Eumkeb G, Chukrathok S. Synergistic activity and mechanism of action of ceftazidime and apigenin combination against ceftazidime-resistant Enterobacter cloacae. Phytomedicine [Internet]. fevereiro de 2013;20(3-4):262-9. Disponível em: https://linkinghub.elsevier.com/retrieve/pii/S0944711312003741

37. Kang SS, Kim J-G, Lee T-H, Oh K-B. Flavonols Inhibit Sortases and SortaseMediated Staphylococcus aureus Clumping to Fibrinogen. Biol Pharm Bull [Internet]. 2006;29(8):1751-5. Disponível em: http://joi.jlc.jst.go.jp/JST.JSTAGE/ $\mathrm{bpb} / 29.1751$ ?from $=$ CrossRef

38. Radovanović B, Anđelković S, Radovanović A, Anđelković M. Antioxidant and Antimicrobial Activity of Polyphenol Extracts from Wild Berry Fruits Grown in Southeast Serbia. Trop J Pharm Res [Internet]. 29 de outubro de 2013;12(5). Disponível em: http://www.ajol.info/index.php/tjpr/article/view/95955

39. Lal K, Yadav P, Kumar A, Kumar A, Paul AK. Design, synthesis, characterization, antimicrobial evaluation and molecular modeling studies of some dehydroacetic acidchalcone-1,2,3-triazole hybrids. Bioorg Chem [Internet]. abril de 2018;77:236-44. Disponível em: https://linkinghub.elsevier.com/retrieve/pii/S0045206817308313

40. Yadav P, Lal K, Kumar L, Kumar A, Kumar A, Paul AK, et al. Synthesis, crystal structure and antimicrobial potential of some fluorinated chalcone-1,2,3-triazole conjugates. Eur J Med Chem [Internet]. julho de 2018;155:263-74. Disponível em: https://linkinghub.elsevier.com/retrieve/pii/S022352341830480X

41. Mori S, Nishizawa N. Methionine as a Dominant Precursor of Phytosiderophores in $<$ italic $>$ Graminaceae $<$ italic $>$ Plants. Plant Cell Physiol [Internet]. setembro de 1987; Disponível em: https://academic.oup.com/pcp/article-lookup/doi/10.1093/ oxfordjournals.pcp.a077388

42. Ayman M, El-Messery SM, Habib EE, Al-Rashood ST, Almehizia AA, Alkahtani HM, et al. Targeting microbial resistance: Synthesis, antibacterial evaluation, DNA binding and modeling study of new chalcone-based dithiocarbamate derivatives. Bioorg Chem [Internet]. abril de 2019;85:282-92. Disponível em: https://linkinghub.elsevier. com/retrieve/pii/S004520681830751X

43. Garrett TPJ, McKern NM, Lou M, Elleman TC, Adams TE, Lovrecz GO, et al. Crystal Structure of a Truncated Epidermal Growth Factor Receptor Extracellular 
Domain Bound to Transforming Growth Factor $\bigotimes$. Cell [Internet]. setembro de 2002;110(6):763-73. Disponível em: https://linkinghub.elsevier.com/retrieve/pii/ S0092867402009406

44. Törmäkangas L, Vuorela P, Saario E, Leinonen M, Saikku P, Vuorela H. In vivo treatment of acute Chlamydia pneumoniae infection with the flavonoids quercetin and luteolin and an alkyl gallate, octyl gallate, in a mouse model. Biochem Pharmacol [Internet]. outubro de 2005;70(8):1222-30. Disponível em: https://linkinghub. elsevier.com/retrieve/pii/S000629520500482X

45. Daglia M. Polyphenols as antimicrobial agents. Curr Opin Biotechnol [Internet]. abril de 2012;23(2):174-81. Disponível em: https://linkinghub.elsevier.com/retrieve/ pii/S0958166911006756

46. Park C, Lee D-G, Kim SW, Choi S-M, Park SH, Chun H-S, et al. Predominance of Community-Associated Methicillin-Resistant Staphylococcus aureus Strains Carrying Staphylococcal Chromosome Cassette mec Type IVA in South Korea. J Clin Microbiol [Internet]. 1 de dezembro de 2007;45(12):4021-6. Disponível em: http:// jcm.asm.org/cgi/doi/10.1128/JCM.01147-07

47. González-Segovia R, Quintanar JL, Salinas E, Ceballos-Salazar R, AvilesJiménez F, Torres-López J. Effect of the flavonoid quercetin on inflammation and lipid peroxidation induced by Helicobacter pylori in gastric mucosa of guinea pig. J Gastroenterol [Internet]. 4 de junho de 2008;43(6):441-7. Disponível em: http://link. springer.com/10.1007/s00535-008-2184-7

48. Isomoto H. Sofalcone, a mucoprotective agent, increases the cure rate of Helicobacter pylori infection when combined with rabeprazole, amoxicillin and clarithromycin. World J Gastroenterol [Internet]. 2005;11(11):1629. Disponível em: http://www.wjgnet.com/1007-9327/full/v11/i11/1629.htm

49. Bernal P, Zloh M, Taylor PW. Disruption of d-alanyl esterification of Staphylococcus aureus cell wall teichoic acid by the $\bigotimes$-lactam resistance modifier (-)-epicatechin gallate. J Antimicrob Chemother [Internet]. junho de 2009;63(6):1156-62. Disponível em: https://academic.oup.com/jac/article-lookup/ doi/10.1093/jac/dkp094

50. Eumkeb G, Sakdarat S, Siriwong S. Reversing $\beta$-lactam antibiotic resistance of Staphylococcus aureus with galangin from Alpinia officinarum Hance and synergism with ceftazidime. Phytomedicine [Internet]. dezembro de 2010;18(1):40-5. Disponível em: https://linkinghub.elsevier.com/retrieve/pii/S0944711310002941

51. Lechner D, Gibbons S, Bucar F. Modulation of isoniazid susceptibility by flavonoids in Mycobacterium. Phytochem Lett. 2008;2:71-5. 
52. Akilandeswari K, Ruckmani K. Synergistic antibacterial effect of apigenin with \-lactam antibiotics and modulation of bacterial resistance by a possible membrane effect against methicillin resistant Staphylococcus aureus. Cell Mol Biol [Internet]. 30 de dezembro de 2016;62(14):74. Disponível em: http://www.cellmolbiol.org/index. $\mathrm{php} / \mathrm{CMB} /$ article/view/1250

53. Qian M, Tang S, Wu C, Wang Y, He T, Chen T, et al. Synergy between baicalein and penicillins against penicillinase-producing Staphylococcus aureus. Int J Med Microbiol [Internet]. setembro de 2015;305(6):501-4. Disponível em: https:// linkinghub.elsevier.com/retrieve/pii/S1438422115000430

54. Usman Amin M, Khurram M, Khan T, Faidah H, Ullah Shah Z, Ur Rahman S, et al. Effects of Luteolin and Quercetin in Combination with Some Conventional Antibiotics against Methicillin-Resistant Staphylococcus aureus. Int J Mol Sci [Internet]. 22 de novembro de 2016;17(11):1947. Disponível em: http://www.mdpi. com/1422-0067/17/11/1947

55. Amin MU, Khurram M, Khattak B, Khan J. Antibiotic additive and synergistic action of rutin, morin and quercetin against methicillin resistant Staphylococcus aureus. BMC Complement Altern Med [Internet]. 12 de dezembro de 2015;15(1):59. Disponível em: http://bmccomplementalternmed.biomedcentral.com/articles/10.1186/ s12906-015-0580-0

56. Cowan MM. Plant Products as Antimicrobial Agents. Clin Microbiol Rev [Internet]. 1 de outubro de 1999;12(4):564-82. Disponível em: http://cmr.asm.org/ lookup/doi/10.1128/CMR.12.4.564

57. Xie Y, Kang R, Sun X, Zhong M, Huang J, Klionsky DJ, et al. Posttranslational modification of autophagy-related proteins in macroautophagy. Autophagy [Internet]. 2 de janeiro de 2015;11(1):28-45. Disponível em: http://www.tandfonline.com/doi/ab s/10.4161/15548627.2014.984267

58. Organização Pan-Americana da Saúde. Resistência aos antibióticos [Internet]. 2017 [citado 25 de junho de 2020]. Disponível em: https://www.paho.org/bra/index. php?option=com_content\&view=article\&id=5664:folha-informativa-resistencia-aosantibioticos\&Itemid $=812$

Autor Correspondente: Mariana Piana

E-mail: marianarpiana@gmail.com Recebido em: 2020-02-27 